\title{
PHENOMENOLOGY OF EXTRA DIMENSIONS
}

\author{
JoAnne L. Hewett \\ Stanford Linear Accelerator Center, Stanford University, Stanford, CA 94309 USA
}

Contributed to Les Houches Summer School on Theoretical Physics: Session 84: Particle Physics Beyond the Standard Model, 08/01/2005--8/26/2005, Les Houches, France 


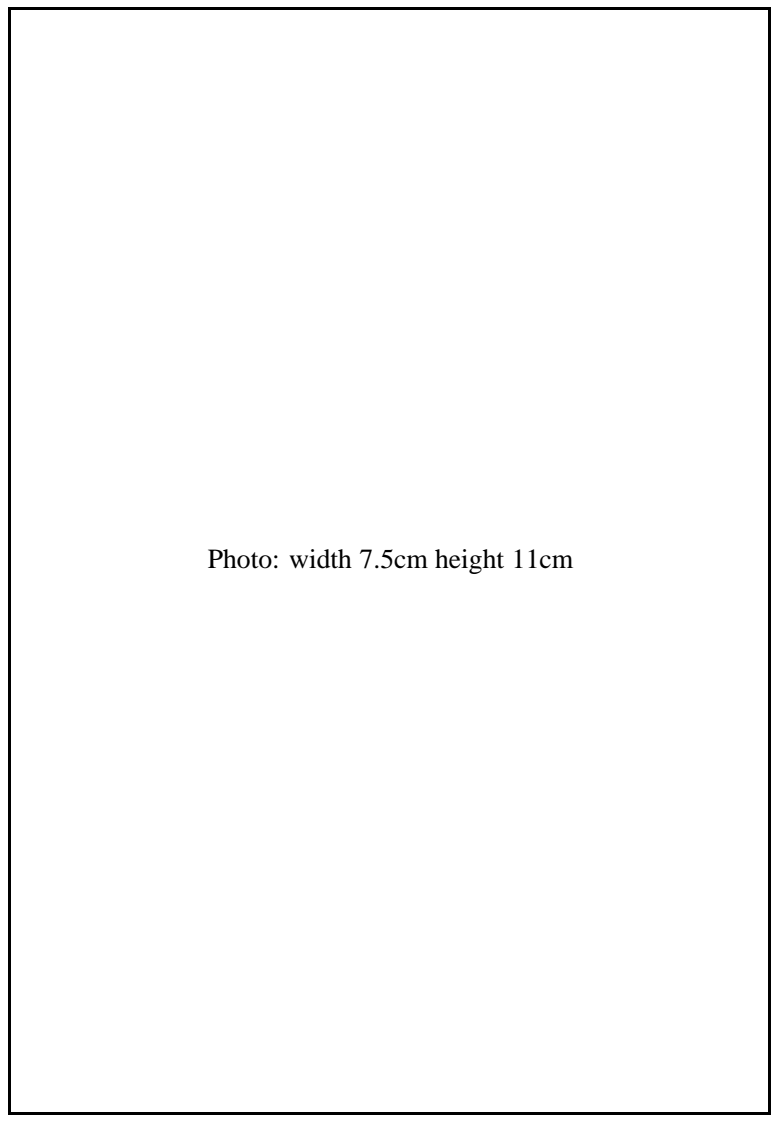




\section{Contents}

1. Introduction 5

2. Large Extra Dimensions $\quad 8$

2.1. Short Range Tests of Gravity 9

2.2. Astrophysical and Cosmological Constraints $\quad 10$

2.3. Collider Probes 15

3. $\mathrm{TeV}^{-}$1-Sized Extra Dimensions 23

4. Warped Extra Dimensions 26

5. Summary 31

References $\quad 32$ 



\section{Introduction}

In particle physics, 4-dimensional Minkowski spacetime is the underlying fundamental framework under which the laws of nature are formulated and interpreted. Relativistic quantum fields exist in spacetime, interactions occur at spacetime points and the laws governing these fields and their interactions are constructed using weighted averages over their spacetime histories. According to the general theory of relativity, fluctuations of the spacetime curvature provide gravitational dynamics. Indeed, experiments show evidence for the predictions of general relativity and hence that spacetime is dynamical at very long length scales. However, gravitational dynamics have yet to be probed at short distances, and it is possible that they are quite different from that implied by a simple extrapolation of the long range theory.

Early attempts to extend general relativity in order to unify gravity and electromagnetism within a common geometrical framework trace back to Gunnar Nordström (1914) [1], Theodor Kaluza (1921) and Oscar Klein (1926) [2]. They proposed that unification of the two forces occurred when spacetime was extended to a five dimensional manifold and imposed the condition that the fields should not depend on the extra dimension. A difficulty with the acceptance of these ideas at the time was a lack of both experimental implications and a quantum description of gravitational dynamics.

Today, one of the most striking requirements of modern string theory, which incorporates both gauge theories and gravitation, is that there must be six or seven extra spatial dimensions. Otherwise the theory is anomalous. Recently, concepts developed within string theory have led to new phenomenological ideas which relate the physics of extra dimensions to observables in a variety of physics experiments.

These new theories have been developed to address the hierarchy problem, i.e., the large disparity between the electroweak scale $\left(\sim 10^{3} \mathrm{GeV}\right)$ where electroweak symmetry breaking occurs and the traditional scale of gravity defined by the Planck scale $\left(10^{19} \mathrm{GeV}\right)$. The source of physics which generates and stabilizes this sixteen order of magnitude difference between the two scales is unknown and represents one of the most puzzling aspects of nature. The novel approach to this long-standing problem proposed in these recent theories is that the geometry of extra spatial dimensions may be responsible for the hierarchy: 
the gravitational field lines spread throughout the full higher dimensional space and modify the behavior of gravity. Indeed, the fact that gravity has yet to be measured at energy scales much above $10^{-3} \mathrm{eV}$ in laboratory experiments admits for the possibility that at higher energies gravity behaves quite differently than expected. The first scenario of this type to be proposed [3] suggested that the apparent hierarchy between these two important scales of nature is generated by a large volume of the extra dimensions, while in a later theoretical framework $[4,5]$ the observed hierarchy results from a strong curvature of the extra dimensional space. If new dimensions are indeed relevant to the source of the hierarchy, then they should provide detectable signatures at the electroweak scale. These physics scenarios with additional dimensions hence afford concrete and distinctive phenomenological predictions for high energy colliders, as well as producing observable consequences for astrophysics and short-range gravity experiments.

Theoretical frameworks with extra dimensions have some general features. In most scenarios, our observed 3-dimensional space is a 3-brane (sometimes called a wall), where the terminology is derived from a generalization of a 2dimensional membrane. This 3-brane is embedded in a higher $D$-dimensional spacetime, $D=3+\delta+1$, with $\delta$ extra spatial dimensions which are orthogonal to our 3-brane. The higher $D$-dimensional space is known as the "bulk". The branes provide a mechanism to hide the existence of extra dimensions in that an observer trapped on a brane can not directly probe the dimensions transverse to the brane without overcoming the brane tension. String theory contains branes upon which particles can be naturally confined or localized [6]. In a general picture, branes carry the Standard Model gauge charges and the ends of open strings are stuck to the branes and represent the Standard Model fields. Fields, such as gravitons, which do not carry Standard Model gauge charges correspond to closed strings and may pop off the brane and propagate throughout the bulk.

The picture is thus one where matter and gauge forces are confined to our 3dimensional subspace, while gravity propagates in a higher dimensional volume. In this case, the Standard Model fields maintain their usual behavior, however, the gravitational field spreads throughout the full $3+\delta$ spatial volume. Conventional wisdom dictates that if the additional dimensions are too large, this would result in observable deviations from Newtonian gravity. The extra dimensional space must then be compactified, i.e, made finite. However, in some alternative theories $[5,7]$, the extra dimensions are infinite and the gravitational deviations are suppressed by other means.

If the additional dimensions are small enough, the Standard Model fields are phenomenologically allowed to propagate in the bulk. This possibility allows for new model-building techniques to address gauge coupling unification [8], supersymmetry breaking $[9,10]$, the neutrino mass spectrum [11], and the fermion 
mass hierarchy [12]. Indeed, the field content which is allowed to propagate in the bulk, as well as the size and geometry of the bulk itself, varies between different models.

As a result of compactification, fields propagating in the bulk expand into a series of states known as a Kaluza-Klein (KK) tower, with the individual KK excitations being labeled by mode numbers. Similar to a particle in a box, the momentum of the bulk field is then quantized in the compactified dimensions. For an observer trapped on the brane, each quanta of momentum in the compactified volume appears as a KK excited state with mass $m^{2}=\vec{p}_{\delta}^{2}$. This builds a KK tower of states, where each state carries identical spin and gauge quantum numbers. If the additional dimensions are infinite instead of being compactified, the $\delta$-dimensional momentum and resulting KK spectrum is continuous.

More technically, in the case where gravity propagates in a compactified bulk, one starts from a $D$-dimensional Einstein-Hilbert action and performs a KK expansion about the metric field of the higher dimensional spacetime. The graviton KK towers arise as a solution to the linearized equation of motion of the metric field in this background [13]. The resulting 4-dimensional fields are the Kaluza-Klein modes. Counting the degrees of freedom within the original higher dimensional metric, the reduction of a spin-2 bulk field results in three distinct classes of towers of KK modes: symmetric tensor, vector fields and scalar fields. The KK zero-mode fields are massless, while the excitation states acquire mass by 'eating' lower spin degrees of freedom. This results in a single 5-component tensor KK tower of massive graviton states, $\delta-1$ gauge KK towers of massive vector states, and $\delta(\delta-1) / 2$ scalar towers. The zero-mode scalar states are radius moduli fields associated with the size of the additional dimensions.

A generalized calculation of the action for linearized gravity in $D$ dimensions can be used to compute the effective 4-dimensional theory. The spin-2 tower of KK states couples to Standard Model fields on the brane via the conserved symmetric stress-energy tensor. The spin-1 KK tower does not induce interactions on the 3-brane. The scalar KK states couple to the Standard Model fields on the brane via the trace of the stress-energy tensor.

The possible experimental signals for the existence of extra dimensions are: (i) the direct or indirect observation of a KK tower of states, or (ii) the observation of deviations in the inverse-square law of gravity in short-range experiments. The detailed properties of the KK states are determined by the geometry of the compactified space and their measurement would reveal the underlying geometry of the bulk.

We now discuss each of the principal scenarios and how they may be probed in experiment. 


\section{Large Extra Dimensions}

The large extra dimensions scenario postulated by Arkani-Hamed, Dimopoulos and Dvali (ADD) [3] makes use of the string inspired braneworld hypothesis. In this model, the Standard Model gauge and matter fields are confined to a 3dimensional brane that exists within a higher dimensional bulk. Gravity alone propagates in the $\delta$ extra spatial dimensions which are compactified. Gauss' Law relates the Planck scale of the effective 4-d low-energy theory, $M_{\mathrm{Pl}}$, to the scale where gravity becomes strong in the $4+\delta$-dimensional spacetime, $M_{D}$, through the volume of the compactified dimensions $V_{\delta}$ via

$$
M_{\mathrm{Pl}}^{2}=V_{\delta} M_{D}^{2+\delta} .
$$

Taking $M_{D} \sim \mathrm{TeV}$, as assumed by $\mathrm{ADD}$, eliminates the hierarchy between $M_{\mathrm{Pl}}$ and the electroweak scale. $M_{\mathrm{Pl}}$ is generated by the large volume of the higher dimensional space and is thus no longer a fundamental scale. The hierarchy problem is now translated to the possibly more tractable question of why the compactification scale of the extra dimensions is large.

If the compactified dimensions are flat, of equal size, and of toroidal form, then $V_{\delta}=\left(2 \pi R_{c}\right)^{\delta}$. For $M_{D} \sim \mathrm{TeV}$, the radius $R_{c}$ of the extra dimensions ranges from a fraction of a millimeter to $\sim 10$ fermi for $\delta$ varying between 2 and 6 . The compactification scale $\left(1 / R_{c}\right)$ associated with these parameters then ranges from $\sim 10^{-4} \mathrm{eV}$ to tens of $\mathrm{MeV}$. The case of one extra dimension is excluded as the corresponding dimension (of size $R_{c} \approx 10^{11} \mathrm{~m}$ ) would directly alter Newton's law at solar-system distances. Our knowledge of the electroweak and strong forces extends with great precision down to distances of order $10^{-15}$ $\mathrm{mm}$, which corresponds to $\sim(100 \mathrm{GeV})^{-1}$. Thus the Standard Model fields do not feel the effects of the large extra dimensions present in this scenario and must be confined to the 3 -brane. Therefore in this model only gravity probes the existence of the extra dimensions ${ }^{1}$.

We now discuss the derivation of the 4-dimensional effective theory, which is computed within linearized quantum gravity. The flat metric is expanded via

$$
G_{A B}=\eta_{A B}+\frac{h_{A B}}{M_{D}^{\delta / 2+1}}
$$

where the upper case indices extend over the full D-dimensional spacetime and $h_{A B}$ represents the bulk graviton fluctuation. The interactions of the graviton are

\footnotetext{
${ }^{1}$ Any Standard Model singlet field, e.g., right handed neutrinos, could also be in the bulk in this scenario.
} 
then described by the action

$$
S_{i n t}=-\frac{1}{M_{D}^{\delta / 2+1}} \int d^{4} x d^{\delta} y_{i} h_{A B}\left(x_{\mu}, y_{i}\right) T_{A B}\left(x_{\mu}, y_{i}\right),
$$

with $T_{A B}$ being the symmetric conserved stress-energy tensor. Upon compactification, the bulk graviton decomposes into the various spin states as described in the introduction and Fourier expands into Kaluza-Klein towers of spin-0, 1, and 2 states which have equally spaced masses of $m_{\vec{n}}=\sqrt{\vec{n}^{2} / R_{c}^{2}}$, where $\vec{n}=$ $\left(n_{1}, n_{2}, \ldots n_{\delta}\right)$ labels the KK excitation level. The spin-1 states do not interact with fields on the 3-brane, the spin-0 states couple to the trace of the stressenergy tensor and will not be considered here. Their phenomenology is described in [14]. Performing the KK expansion for the spin-2 tower, setting $T_{A B}=\eta_{A}^{\mu} \eta_{B}^{\nu} T_{\mu \nu} \delta\left(y_{i}\right)$ for the Standard Model fields confined to the brane, and integrating the action over the extra dimensional coordinates $y_{i}$ gives the interactions of the graviton KK states with the Standard Model fields. All the states in the KK tower, including the $\vec{n}=0$ massless state, couple in an identical manner with universal strength of $M_{\mathrm{Pl}}^{-1}$. The corresponding Feynman rules are catalogued in $[15,16]$.

The existence of large extra dimenions would affect a broad range of physical processes. Their presence may be detected in tests of short range gravity, astrophysical considerations, and collider experiments. We now review each of these in turn.

\subsection{Short Range Tests of Gravity}

Until very recently, the inverse square force law of Newtonian gravity had been precisely tested only down to distances of order a centimeter $[17,18]$. Such tests are performed by short range gravity experiments that probe new interactions by searching for deviations from Newtonian gravity at small distances. There are several parameterizations which describe these potential deviations [19]; the one most widely used by experiments is that where the classical gravitational potential is expanded to include a Yukawa interaction:

$$
V(r)=-\frac{1}{M_{\mathrm{Pl}}^{2}} \frac{m_{1} m_{2}}{r}\left(1+\alpha e^{-r / \lambda}\right) .
$$

Here, $r$ is the distance between two masses $m_{1}$ and $m_{2}$ and is fixed by the experimental apparatus, $\alpha$ is a dimensionless parameter relating the strength of the additional Yukawa interaction to that of gravity, and $\lambda$ is the range of the new interaction. The best experimental sensitivity is achieved for the case $\lambda \approx r$, with the sensitivity decreasing rapidly for smaller distances. The experimental results are presented in the $\alpha-\lambda$ plane in the form of convex curves that are 
centered around the distance at which a particular experiment operates. These short range tests are performed by Van-der-Waals (probing $1 / r^{3}$ deviations) and Casimir (probing $1 / r^{4}$ terms) force experiments, as well as Cavendish-type detectors which directly measure the gravitational force.

The two-body potential given by Gauss' Law in the presence of additional dimensions (for distances $r<R_{c}$ ) is expressed as [3]:

$$
V(r)=-\frac{1}{8 \pi M_{D}^{2+\delta}} \frac{m_{1} m_{2}}{r^{\delta+1}}
$$

in the conventions of [15], which we employ throughout these lectures. When the two masses are separated by a distance $r>R_{c}$ and the dimensions are assumed to be compactified on a torus of radius $R_{c}$ the potential becomes:

$$
V(r)=-\frac{1}{8 \pi M_{D}^{2+\delta}} \frac{m_{1} m_{2}}{R_{c}^{\delta}} \frac{1}{r},
$$

i.e., the usual $1 / r$ Newtonian potential is recovered using Gauss' Law. The parameters in the general form of the two-body potential in Eq. (2.4), i.e., $\alpha$ and $\lambda$, depend on the number of extra dimensions and the type of compactification [20]; for the simple case of compactifying on a torus, the range $\lambda$ of the new interaction is the compactification radius $R_{c}$, and $\alpha=2 \delta$. It should be noted that the dependence of Eq. (2.6) on $M_{D}$ is related to the compactification scheme through the precise form of the volume factor.

The most recent Cavendish-type experiment [21] used a torsion pendulum and a rotating attractor. These results are displayed as the curve labeled Eöt-Wash in Figure 1. Interpreted within the framework of two large additional spatial dimensions, the results imply that $R_{c}<130 \mu \mathrm{m}$. The relation of this bound to the fundamental scale $M_{D}$ depends on the compactification scheme. For $\delta>2$, $R_{c}$ is too small for the effects of extra dimensions to be probed in mechanical experiments. Results from other searches are also shown in the $\alpha-\lambda$ plane in this figure. The predictions and allowed regions in the $\alpha-\lambda$ parameter space from other theoretical considerations are also presented in the figure; they include scenarios with axions, dilatons and scalar moduli fields from string theory, and attempted solutions to the cosmological constant problem.

\subsection{Astrophysical and Cosmological Constraints}

Astrophysical and cosmological considerations impose strict constraints on some theories of extra dimensions; in particular, early universe cosmology can be drastically altered from the standard picture. The typical energy scale associated with such considerations is of order $100 \mathrm{MeV}$, and models with $\mathrm{KK}$ states that can be produced in this energy regime are highly restricted. 


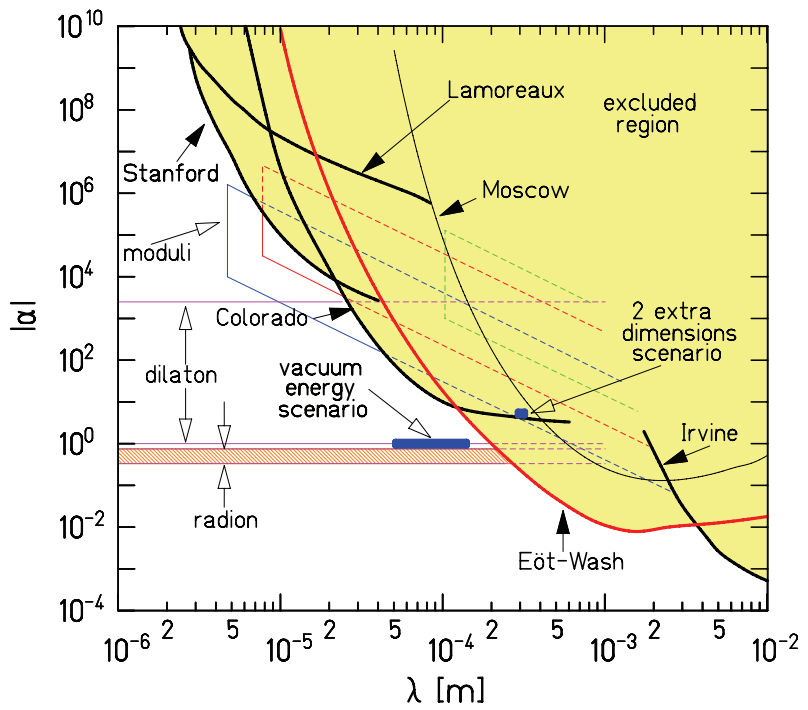

Fig. 1. 95\% confidence level upper limits on the strength $\alpha$ (relative to Newtonian gravity) as a function of the range $\lambda$ of additional Yukawa interactions. The region excluded by previous experiments [18] lies above the curves labeled Irvine, Moscow and Lamoreaux. The most recent results [21] correspond to the curves labeled Eöt-Wash. 
For the case of large extra dimensions of flat and toroidal form, the astrophysical bounds far surpass those from collider or short range gravity experiments for $\delta=2$. If these large additional dimensions are compactified on a hyperbolic manifold instead, then the astrophysical constraints are avoided [22] as the modified spectrum of KK graviton states admits for a first excitation mass of order several GeV. Alternatively, these bounds are also weakened if a Ricci term is present on the brane since that serves to suppress graviton emission rates [23].

We now describe the various astrophysical and cosmological considerations that restrict the scenario with large flat extra dimensions. These processes include graviton emission during the core collapse of supernovae, the heating of neutron stars from graviton decays, considerations of the cosmic diffuse $\gamma$-ray background, overclosure of the universe, matter dominated cooling of the universe, and reheating of the universe. The restrictions obtained from processes that include effects from the decays of KK states rely on the assumption that the KK modes can only decay into Standard Model particles on one brane, i.e., there are no additional branes in the theory, and that decays into other KK modes with smaller bulk momenta do not occur.

During the core collapse of type II supernova (SN), most of the gravitational binding energy is radiated by neutrinos. This hypothesis has been confirmed by measurements of neutrino fluxes from SN1987A by the Kamiokande and IMB collaborations [24]. Any light, neutral, weakly interacting particle which couples to nucleons, such as bulk gravitons, will compete with neutrinos in carrying energy away from the stellar interior. The rate at which the supernova core can lose energy through emission of KK states can then be used to constrain the fundamental scale $M_{D}[25,26]$. The graviton emission process is nucleon 'gravisstrahlung', $N+N \rightarrow N+N+X$, where $N$ can be a proton or neutron, and $X$ represents the contributions from massive KK graviton states, ordinary gravitons, and the KK dilaton (scalar) modes which are a remnant of the bulk graviton decomposition. If present, this gravisstrahlung process would provide an additional heat sink and accelerate the supernova cooling in violation with the observations of SN1987A. This process is highly dependent on the temperature of the core at collapse, which is estimated to be $T \approx 30-70 \mathrm{MeV}$, and on the core density, $\rho \approx(3-10) \times 10^{14} \mathrm{gcm}^{-3}$. Several additional uncertainties, such as the form of the nucleon scattering matrix, the specific heat of matter at high density, and the neutrino transport mean-free path at high density, also enter the calculation. These uncertainties are computed using the well studied nucleon-nucleon axion bremsstrahlung process. The most conservative constraint on KK emission [26] yields $R_{c} \leq 7.1 \times 10^{-4} \mathrm{~mm}$ for $\delta=2$ and $R_{c} \leq 8.5 \times 10^{-7} \mathrm{~mm}$ for $\delta=3$, taking $T_{S N 1987 A}=30 \mathrm{MeV}$.

A complementary bound arises from the radiative decay of the Kaluza Klein gravitons produced by the core collapse of all supernovae that have exploded 
during the history of the universe ( $\mathrm{SNe}$ ). The two photon decay mode is kinematically favored for the lower mass KK modes [16] with this lifetime being $\tau_{\gamma \gamma} \approx 3 \times 10^{9} \mathrm{yr}\left(\frac{100 \mathrm{MeV}}{\mathrm{m}_{\mathrm{KK}}}\right)^{3}$. Over the age of the universe, a significant fraction of the KK states emitted from supernovae cores will have decayed into photons, contributing to the cosmic diffuse $\gamma$-ray background. This is estimated using the present day supernova rate and the gravisstrahlung rate discussed above. A bound on the size of the additional dimensions is then imposed from the measured cosmic $\gamma$-ray background. For a choice of cosmological parameters the predicted $\gamma$-flux exceeds the observations by EGRET or COMPTEL [28] unless the fraction of the SN energy released via gravisstrahlung is less than about $0.5-1 \%$ of the total. For two extra dimensions, the limit on the compactification radius is $R_{c} \leq 0.910^{-4} \mathrm{~mm}$ and for three extra dimensions the bound is $R_{c} \leq 0.1910^{-6}$ $\mathrm{mm}$ [27]. Additional contributions to the cosmic diffuse $\gamma$-ray background arise when the KK gravitons are produced from other sources such as neutrino annihilation, $\nu \bar{\nu} \rightarrow G_{n} \rightarrow \gamma \gamma$. These were considered in [29], and by placing a bound on the normalcy temperature required by Big Bang Nucleosynthesis the limit $R_{c} \leq 5.1 \times 10^{-5} \mathrm{~mm}$ for $\delta=2$ is obtained.

In [29], it is assumed that the universe enters the radiation dominated epoch instantaneously at the reheating temperature. However, it is plausible that the universe enters the radiation epoch after being reheated by the decay of a massive scalar field or by some other means of entropy production. If a large number of KK states are produced during reheating, they are non-relativistic and hence are not diluted by entropy production. Their subsequent decays contribute to the diffuse $\gamma$-ray background. Using data from COMPTEL and EGRET [28], the constraints on $M_{D}$ are tightened and are 167, 21.7, 4.75, and $1.55 \mathrm{TeV}$ for $\delta=2,3,4$, and 5 , respectively, assuming that a $1 \mathrm{GeV}$ maximum temperature is reached during reheating [30].

The escape velocity of a neutron star is similar to the average speed of thermally produced KK states in a SN core collapse, and hence a large fraction of the KK states can become trapped within the core halo. The decays of these states will continue to be a source of $\gamma$-rays long after the SN explosion. Comparisons of the expected contributions to the $\gamma$-ray flux rate from this source with EGRET data [28] from nearby neutron stars and pulsars constrains [31] the fraction of the SN energy released via gravisstrahlung to be less than about $10^{-5}$ of the total. For two extra dimensions this yields the bound $M_{D} \gtrsim 450 \mathrm{TeV}$ and for $\delta=3$ the constraint is $M_{D} \gtrsim 30 \mathrm{TeV}$. The expected sensitivity from GLAST [32] will increase these limits by a factor of 2 to 3 .

The Hubble space telescope has observed that the surface temperature of several older neutron stars is higher than that expected in standard cooling models. A possible source for this excess heat is the decays of the KK graviton states 
trapped in the halo surrounding the star. The $\gamma$ 's, electrons, and neutrinos from the KK decays then hit the star and heat it. For the estimated heating rate from this mechanism not to exceed the observed luminosity, the fraction of the SN energy released via gravisstrahlung must be $\lesssim 5 \times 10^{-8}$ of the total [31], with the exact number being uncertain by a factor of a few due to theoretical and experimental uncertainties. This is by far the most stringent constraint yielding $M_{D} \gtrsim 1700,60 \mathrm{TeV}$ for $\delta=2,3$, respectively. Although the calculations for SN emissions have not been performed for $\delta>4$, simple scaling suggests that this mechanism results in $M_{D} \gtrsim 4,0.8 \mathrm{TeV}$ for $\delta=4,5$, respectively.

Once produced, the massive KK gravitons are sufficiently long-lived as to potentially overclose the universe. Comparisons of $\mathrm{KK}$ graviton production rates from photon, as well as neutrino, annihilation to the critical density of the universe results [29] in $R_{c}<1.5 h \times 10^{-5} \mathrm{~m}$ for 2 additional dimensions, where $h$ is the current Hubble parameter in units of $100 \mathrm{~km} / \mathrm{sMpc}$. While this constraint is milder than those obtained above, it is less dependent on assumptions regarding the existence of additional branes.

Overproduction of Kaluza Klein modes in the early universe could initiate an early epoch of matter radiation equality which would lead to a too low value for the age of the universe. For temperatures below $\sim 100 \mathrm{MeV}$, the cooling of the universe can be accelerated by $\mathrm{KK}$ mode production and evaporation into the bulk, as opposed to the normal cosmological expansion. Using the present temperature of the cosmic microwave background of $2.73 \mathrm{~K}\left(=2.35 \times 10^{-10}\right.$ $\mathrm{MeV})$ and taking the minimum age of the universe to be 12.8 Gyrs $(=6.2 \times$

$10^{39} \mathrm{MeV}^{-1}$ ), as determined by the mean observed age of globular clusters, a maximum temperature can be imposed at radiation-matter equality which cannot be exceeded by the overproduction of KK modes at early times. The resulting lower bounds are $M_{D}$ are $86,7.4$, and $1.5 \mathrm{TeV}$ for $\delta=2,3$, and 4 respectively [33]. Further considerations of the effects from overproduction of KK states on the characteristic scale of the turn-over of the matter power spectra at the epoch of matter radiation equality show that the period of inflation must be extended down to very low temperatures in order to be consistent with the latest data from galaxy surveys [34].

We collect the constraints from these considerations in Table 1, where we state the restrictions in terms of bounds on the fundamental scale $M_{D}$. We note that the relation of the above constraints to $M_{D}$ is tricky as numerical conventions, as well as assumptions regarding the compactification scheme, explicitly enter some of the computations; in particular, that of gravisstrahlung production during supernova collapse. In addition, all of these bounds assume that all of the additional dimensions are of the same size. The constraints in the table are thus merely indicative and should not be taken as exact.

To conclude this section, we discuss the possible contribution of graviton $\mathrm{KK}$ 


\begin{tabular}{|l|c|c|c|c|}
\hline & \multicolumn{4}{|c|}{$\delta$} \\
\hline Supernova Cooling [26] & 2 & 3 & 4 & 5 \\
Cosmic Diffuse $\gamma$-Rays: & 30 & 2.5 & & \\
$\quad$ Cosmic SNe [27] & 80 & 7 & & \\
$\nu \bar{\nu}$ Annihilation [29] & 110 & 5 & & \\
Re-heating [30] & 170 & 20 & 5 & 1.5 \\
Neutron Star Halo [31] & 450 & 30 & & \\
Overclosure of Universe [29] & $6.5 / \sqrt{h}$ & & & \\
Matter Dominated Early Universe [33] & 85 & 7 & 1.5 & \\
Neutron Star Heat Excess [31] & 1700 & 60 & 4 & 1 \\
\hline \hline
\end{tabular}

Table 1

Summary of constraints on the fundamental scale $M_{D}$ in TeV from astrophysical and cosmological considerations as discussed in the text.

states to the production of high-energy cosmic rays beyond the GZK cut-off of $10^{20} \mathrm{eV}$. About 20 super-GZK events have been observed and their origin is presently unknown. In the case of large extra dimensions, KK graviton exchange can contribute to high-energy $\nu$-nucleon scattering and produce hadronic sized cross sections above the GZK cut-off for $M_{D}$ in the range of 1 to $10 \mathrm{TeV}$ [35].

\subsection{Collider Probes}

If such additional dimensions are present and quantum gravity becomes strong at the $\mathrm{TeV}$ scale, then observable signatures at colliders operating at $\mathrm{TeV}$ energies must be induced.

One may wonder how interactions of this type can be observable at colliders since the coupling strength is so weak. In the ADD scenario, there are $\left(E R_{c}\right)^{\delta}$ massive Kaluza-Klein modes that are kinematically accessible in a collider process with energy $E$. For $\delta=2$ and $E=1 \mathrm{TeV}$, that totals $10^{30}$ graviton KK states which may individually contribute to a process. It is the sum over the contribution from each KK state which removes the Planck scale suppression in a process and replaces it by powers of the fundamental scale $M_{D} \sim \mathrm{TeV}$. The interactions of the massive Kaluza-Klein graviton modes can then be observed in collider experiments either through missing energy signatures or through their virtual exchange in Standard Model processes. At future colliders with very high energies, it is possible that quantum gravity phenomena are accessible resulting in explicit signals for string or brane effects; these will be discussed briefly in Section 5. We now discuss in detail the two classes of collider signatures for large extra dimensions.

The first class of collider processes involves the real emission of Kaluza-Klein 
graviton states in the scattering processes $e^{+} e^{-} \rightarrow \gamma(Z)+G_{n}$, and $p \bar{p} \rightarrow g+G_{n}$, or in $Z \rightarrow f \bar{f}+G_{n}$. The produced graviton behaves as if it were a massive, noninteracting, stable particle and thus appears as missing energy in the detector. The cross section is computed for the production of a single massive KK excitation and then summed over the full tower of KK states. Since the mass splittings between the KK states is so small, the sum over the states may be replaced by an integral weighted by the density of KK states. The specific process kinematics cut off this integral, rendering a finite and model independent result. The expected suppression from the $M_{\mathrm{Pl}}^{-1}$ strength of the graviton KK couplings is exactly compensated by a $M_{\mathrm{Pl}}^{2}$ enhancement in the phase space integration. The cross section for on-shell production of massive Kaluza Klein graviton modes then scales as simple powers of $\sqrt{s} / M_{D}$,

$$
\sigma_{K K} \sim \frac{1}{M_{\mathrm{Pl}}^{2}}\left(\sqrt{s} R_{c}\right)^{\delta} \sim \frac{1}{M_{D}^{2}}\left(\frac{\sqrt{s}}{M_{D}}\right)^{\delta}
$$

The exact expression may be found in $[15,36]$. It is important to note that due to integrating over the effective density of states, the radiated graviton appears to have a continuous mass distribution; this corresponds to the probability of emitting gravitons with different extra dimensional momenta. The observables for graviton production, such as the $\gamma / Z$ angular and energy distributions in $e^{+} e^{-}$ collisions, are then distinct from those of other physics processes involving fixed masses for the undetectable particles. In particular, the SM background is given by the 3-body production $e^{+} e^{-} \rightarrow \nu \bar{\nu} \gamma$.

The cross section for $e^{+} e^{-} \rightarrow \gamma G_{n}$ as a function of the fundamental Planck scale is presented in Fig. 2 for $\sqrt{s}=1 \mathrm{TeV}$. The level of SM background is also shown, with and without electron beam polarization set at $90 \%$. We note that the signal(background) increases(decreases) with increasing $\sqrt{s}$. Details of the various distributions associated with this process can be found in Ref. [15].

Searches for direct KK graviton production in the reaction $e^{+} e^{-} \rightarrow G_{n}+$ $\gamma(Z)$ at LEP II, using the characteristic final states of missing energy plus a single photon or $\mathrm{Z}$ boson, have excluded [37] fundamental scales up to $\sim 1.45 \mathrm{TeV}$ for two extra compactified dimensions and $\sim 0.6 \mathrm{TeV}$ for six extra dimensions. These analyses use both total cross section measurements and fits to angular distributions to set a limit on the graviton production rates as a function of the number of extra dimensions. The expected discovery reach from this process has been computed in Ref. [38] at a high energy linear $e^{+} e^{-}$collider with $\sqrt{s}=800$ $\mathrm{GeV}, 1000 \mathrm{fb}^{-1}$ of integrated luminosity, and various configurations for the beam polarization. These results are displayed in Table 2 and include kinematic acceptance cuts, initial state radiation, and beamsstrahlung. 


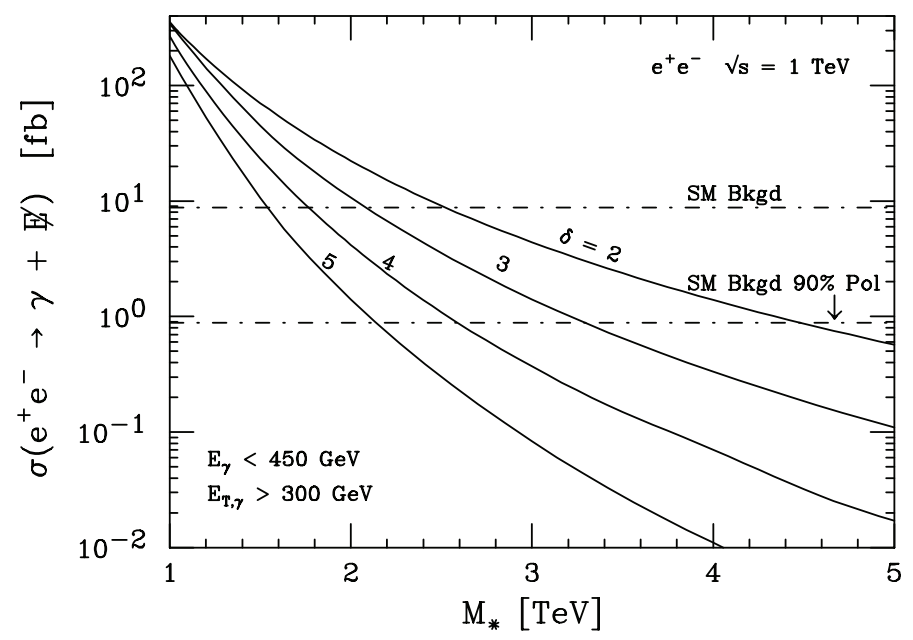

Fig. 2. The cross section for $e^{+} e^{-} \rightarrow \gamma G_{n}$ for $\sqrt{s}=1 \mathrm{TeV}$ as a function of the fundamental Planck scale for various values of $\delta$ as indicated. The cross section for the SM background, with and without $90 \%$ beam polarization correspond to the horizontal lines as labeled. From [15].

The emission process at hadron colliders, for example, $q \bar{q} \rightarrow g+G_{n}$, results in a monojet plus missing transverse energy signature. For larger numbers of extra dimenisons the density of the KK states increases rapidly and the KK mass distribution is shifted to higher values. This is not reflected in the missing energy distribution: although the heavier KK gravitons are more likely to carry larger energy, they are also more likely to be produced at threshold due to the rapidly decreasing parton distribution functions. These two effects compensate each other, leaving nearly identical missing energy distributions. In addition, the effective low-energy theory breaks down for some regions of the parameter space as the parton-level center of mass energy can exceed the value of $M_{D}$. Experiments are then sensitive to the new physics appearing above $M_{D}$ that is associated with the low-scale quantum gravity.

Searches from the Tevatron Run I yield similar results as those from LEP II, and it is anticipated that Run II at the Tevatron will have a higher sensitivity [39]. An ATLAS simulation [40] of the missing transverse energy in signal and background events at the LHC with $100 \mathrm{fb}^{-1}$ is presented in Fig. 3 for various values of $M_{D}$ and $\delta$. This study results in the discovery range displayed in Table 2. The lower end of the range corresponds to where the ultraviolet physics sets in and the effective theory fails, while the upper end represents the boundary where the signal is observable above background. 


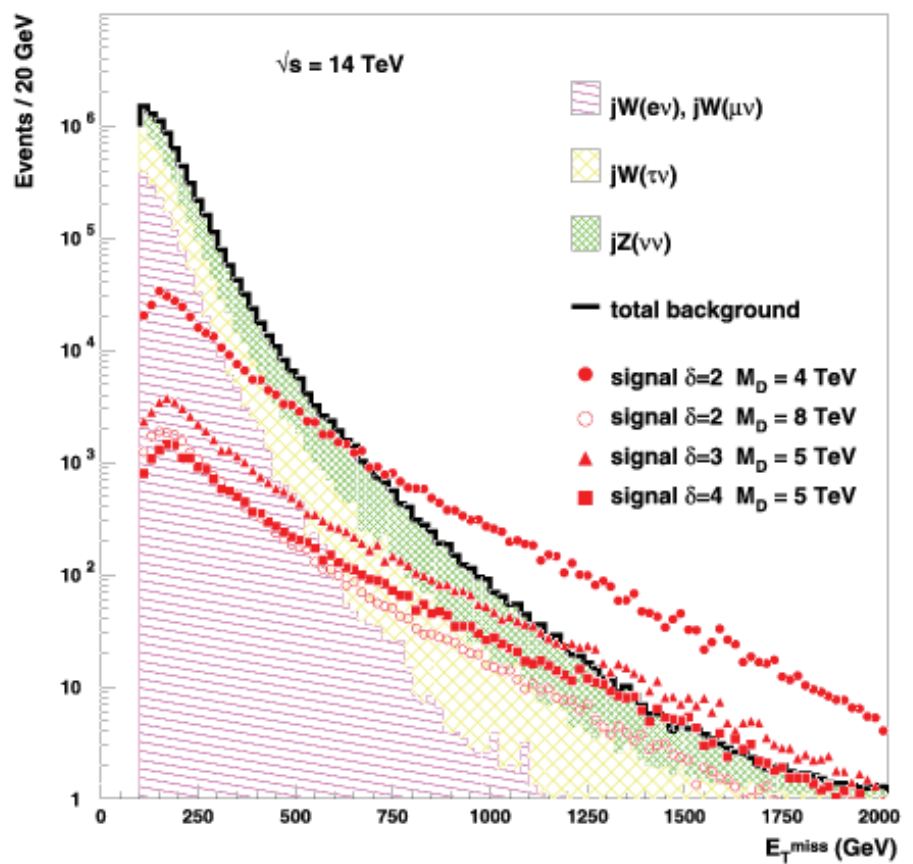

Fig. 3. Distribution of the missing transverse energy in background events and signal events for 100 $\mathrm{fb}^{-1}$. The contribution of the three principal Standard Model background processes is shown as well as the distribution of the signal for several values of $\delta$ and $M_{D}$. From [40]. 


\begin{tabular}{|c|l|c|c|c|}
\hline \hline$e^{+} e^{-} \rightarrow \gamma+G_{n}$ & & 2 & 4 & 6 \\
\hline LC & $P_{-,+}=0$ & 5.9 & 3.5 & 2.5 \\
LC & $P_{-}=0.8$ & 8.3 & 4.4 & 2.9 \\
LC & $P_{-}=0.8, P_{+}=0.6$ & 10.4 & 5.1 & 3.3 \\
$p p \rightarrow g+G_{n}$ & & 2 & 3 & 4 \\
\hline LHC & & $4-8.9$ & $4.5-6.8$ & $5.0-5.8$ \\
\hline
\end{tabular}

Table 2

95\% CL sensitivity to the fundamental scale $M_{D}$ in TeV for different values of $\delta$, from the emission process for various polarization configurations and different colliders as discussed in the text. $\sqrt{s}=$ $800 \mathrm{GeV}$ and $1 \mathrm{ab}^{-1}$ has been assumed for the LC and $100 \mathrm{fb}^{-1}$ for the LHC. Note that the LHC only probes $M_{D}$ within the stated range.

If an emission signal is observed, one would like to determine the values of the fundamental parameters, $M_{D}$ and $\delta$. The measurement of the cross section at a linear collider at two different values of $\sqrt{s}$ can be used to determine these parameters [38] and test the consistency of the data with the large extra dimensions hypothesis. This is illustrated in Fig. 4.

Lastly, we note that the cross section for the emission process can be reduced somewhat if the 3-brane is flexible, or soft, instead of being rigid [41]. In this case, the brane is allowed to recoil when the KK graviton is radiated; this can be parameterized as an exponential suppression of the cross section, with the exponential being a function of the brane tension $\Delta_{\tau}$

$$
\frac{d \sigma}{d x_{\gamma} d \cos \theta}(\text { soft })=\frac{d \sigma}{d x_{\gamma} d \cos \theta}(\text { stiff }) e^{s\left(1-x_{\gamma}\right) / \Delta_{\tau}^{2}},
$$

where $x_{\gamma}$ is the scaled energy of the photon. For reasonable values of the brane tension, this suppression is not numerically large. The brane tension can also be determined by mapping out the cross section as a function of $\sqrt{s}$ as shown in Fig. 4

The second class of collider signals for large extra dimensions is that of virtual graviton exchange $[15,42]$ in $2 \rightarrow 2$ scattering. This leads to deviations in cross sections and asymmetries in Standard Model processes, such as $e^{+} e^{-} \rightarrow f \bar{f}$. It may also give rise to new production processes which are not present at treelevel in the Standard Model, such as $g g \rightarrow \ell^{+} \ell^{-}$. The signature is similar to that expected in composite theories and provides a good experimental tool for searching for large extra dimensions for the case $\sqrt{s}<M_{D}$.

Graviton exchange is governed by the effective Lagrangian

$$
\mathcal{L}=i \frac{4 \lambda}{\Lambda_{H}^{4}} T_{\mu \nu} T^{\mu \nu}+\text { h.c. }
$$



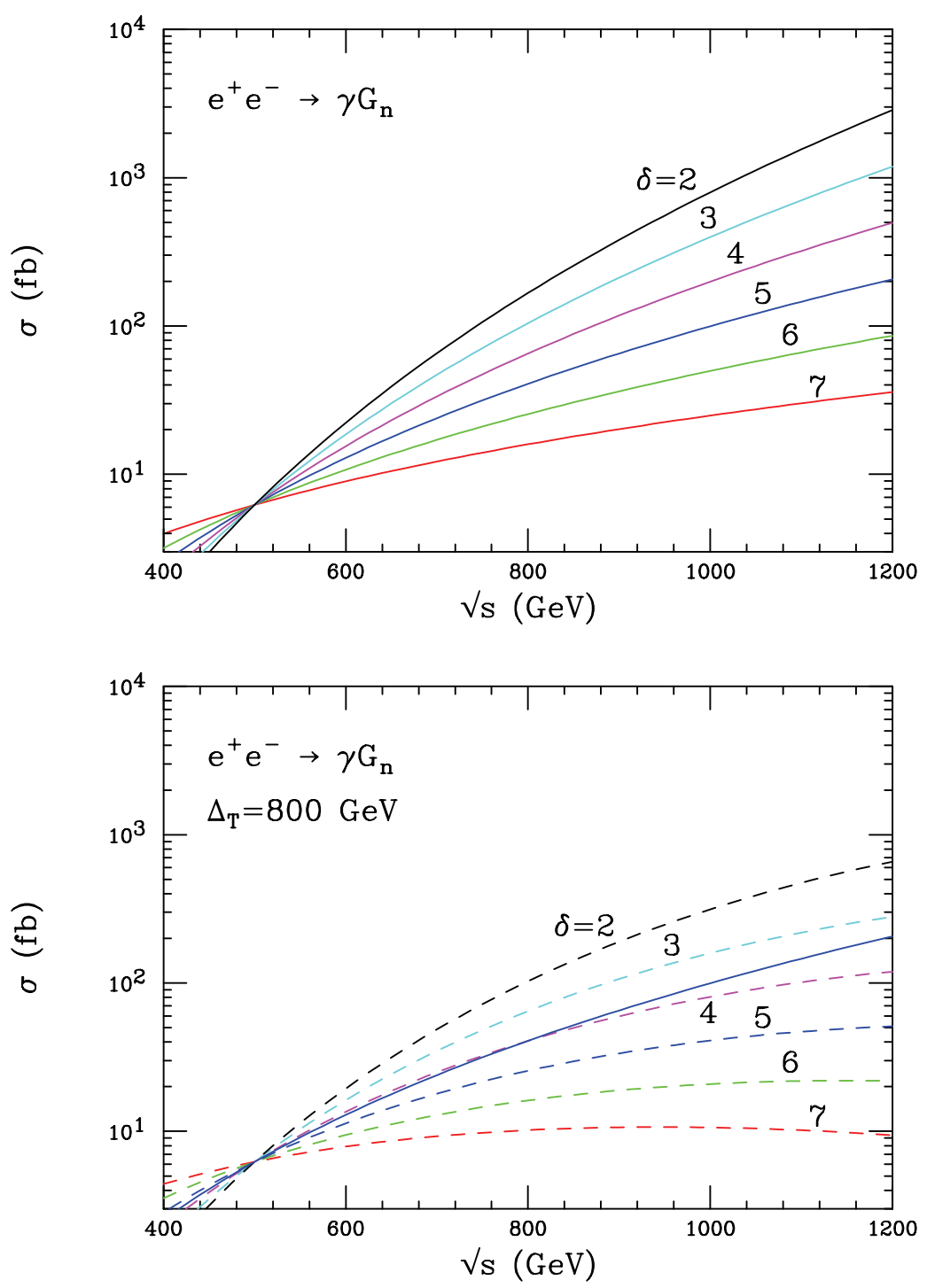

Fig. 4. Emission cross section in $e^{+} e^{-}$annihilation as a function of $\sqrt{s}$ for $\delta=2-7$ from bottom to top on the right-hand side. The cross sections are normalized to $M_{D}=5 \mathrm{TeV}$ and $\delta=2$ at $\sqrt{s}=500 \mathrm{GeV}$. Top" Brane terms are not included. Bottom: The effects of finite brane tension are included, taking the relevant tension parameter to be $\Delta_{\tau}=800 \mathrm{GeV}$. Here, the lone solid curve represents the case without brane term effects for $\delta=5$. 
The amplitude is proportional to the sum over the propagators for the graviton KK tower which may be converted to an integral over the density of KK states. However, in this case, there is no specific cut-off associated with the process kinematics and the integral is divergent for $\delta>1$. This introduces a sensitivity to the unknown ultraviolet physics which appears at the fundamental scale. This integral needs to be regulated and several approaches have been proposed: (i) a naive cut-off scheme [15,42] (ii) brane fluctuations [43], or (iii) the inclusion of full weakly coupled $\mathrm{TeV}$-scale string theory in the scattering process $[39,44]$. The most model independent approach which does not make any assumptions as to the nature of the new physics appearing at the fundamental scale is that of the naive cut-off. Here, the cut-off is set to $\Lambda_{H} \neq M_{D}$; the exact relationship between $\Lambda_{H}$ and $M_{D}$ is not calculable without knowledge of the full theory. The parameter $\lambda= \pm 1$ is also usually incorporated in direct analogy with the standard parameterization for contact interactions [45] and accounts for uncertainties associated with the ultraviolet physics. The substitution

$$
\mathcal{M} \sim \frac{i^{2} \pi}{M_{\mathrm{Pl}}^{2}} \sum_{\vec{n}=1}^{\infty} \frac{1}{s-m_{\vec{n}}^{2}} \rightarrow \frac{\lambda}{\Lambda_{H}^{4}}
$$

is then performed in the matrix element for s-channel KK graviton exchange with corresponding replacements for $\mathrm{t}$ - and $\mathrm{u}$-channel scattering. As above, the Planck scale suppression is removed and superseded by powers of $\Lambda_{H} \sim \mathrm{TeV}$.

The resulting angular distributions for fermion pair production are quartic in $\cos \theta$ and thus provide a unique signal for spin- 2 exchange. An illustration of this is given in Fig. 5 which displays the angular dependence of the polarized Left-Right asymmetry in $e^{+} e^{-} \rightarrow b \bar{b}$.

The experimental analyses also make use of the cut-off approach. Using virtual Kaluza-Klein graviton exchange in reactions with diphoton, diboson and dilepton final states, $e^{+} e^{-} \rightarrow G_{n} \rightarrow \gamma \gamma, V V$, $\ell \ell$, LEP experiments [46] exclude $\Lambda_{H} \lesssim 0.5-1.0 \mathrm{TeV}$ independent of the number of extra dimensions. At the Tevatron [47], the combined Drell-Yan and diphoton channels exclude exchange scales up to $\sim 1.1 \mathrm{TeV}$. In addition, $\mathrm{H} 1$ and ZEUS at HERA [48] have both placed the bound $\Lambda_{H} \gtrsim 800 \mathrm{GeV}$.

The potential search reach for virtual KK graviton exchange in processes at future accelerators are listed in Table 3. These sensitivities are estimated for the LHC [49], a high energy $e^{+} e^{-}$linear collider [42], as well as for a $\gamma \gamma$ collider [50], where the initial photon beams originate from Compton laser backscattering. Note that the $\gamma \gamma \rightarrow W W$ process has the highest sensitivity to graviton exchange.

In summary, present facilities have searched for large extra dimensions and excluded their existence for fundamental scales up to $\sim \mathrm{TeV}$. The reach of future 


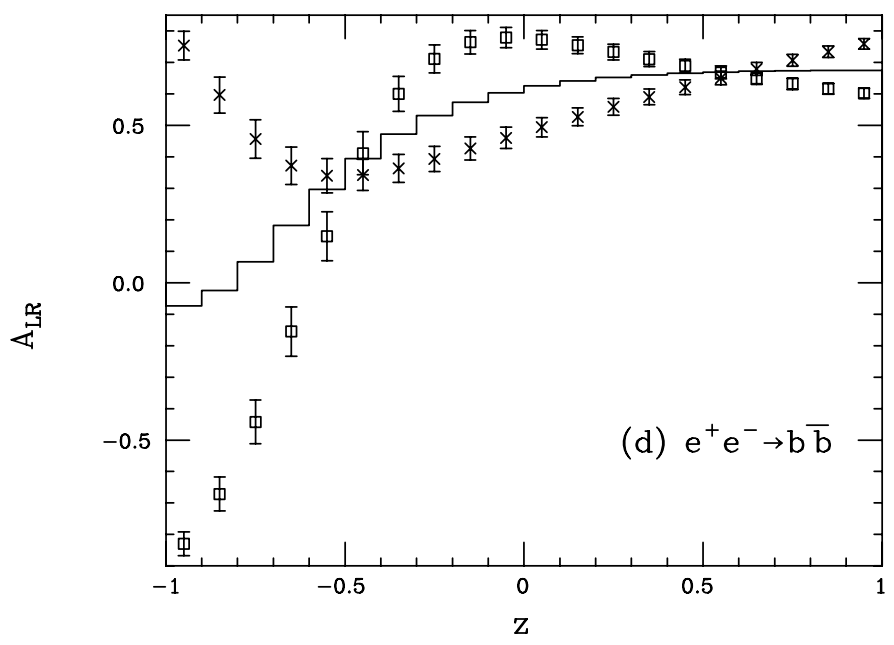

Fig. 5. Distribution of the angular dependence $(z=\cos \theta)$ of the polarized Left-Right asymmetry in $e^{+} e^{-} \rightarrow b \bar{b}$ with $\sqrt{s}=500 \mathrm{GeV}$, taking $M_{D}=1.5 \mathrm{TeV}$ and $\lambda= \pm 1$. The solid histogram is the Standard Model expectation. The two sets of data points correspond to the two choices of sign for $\lambda$, and the error bars represent the statistics in each bin for an integrated luminosity of $75 \mathrm{fb}^{-1}$. From [42].

\begin{tabular}{|c|c|c|c|}
\hline & & $\sqrt{s}(\mathrm{TeV})$ & $M_{H}(\mathrm{TeV})$ \\
\hline \multirow{5}{*}{$\mathrm{LC}$} & $e^{+} e^{-} \rightarrow f \bar{f}$ & 0.5 & 4.1 \\
& $e^{+} e^{-} \rightarrow f \bar{f}$ & 1.0 & 7.2 \\
& $\gamma \gamma \rightarrow \gamma \gamma$ & 1.0 & 3.5 \\
& $\gamma \gamma \rightarrow W W$ & 1.0 & 13.0 \\
& $e \gamma \rightarrow e \gamma$ & 1.0 & 8.0 \\
LHC & $p p \rightarrow \ell^{+} \ell^{-}$ & 14.0 & 7.5 \\
& $p p \rightarrow \gamma \gamma$ & 14.0 & 7.1 \\
\hline \hline
\end{tabular}

Table 3

The estimated $95 \%$ CL search reach for $M_{H}$ from various processes at future accelerators. 
facilities will extend this reach to a sensitivity of $\sim 10 \mathrm{TeV}$. If this scenario is indeed relevant to the hierarchy, then it should be discovered in the next round of experiments. In addition, future experiments will have the capability to determine the geometry of the higher dimensional space, such as the size and number of extra dimensions, as well as the degree of the brane tension.

If the fundamental scale of gravity is at roughly a $\mathrm{TeV}$, then future colliders will directly probe new exotic degrees of freedom in addition to the Kaluza Klein modes of extra dimensions, including the effects of quantum gravity itself. We do not yet have unambiguous predictions for this new and unknown physics, but it could take the form of new strongly interacting gauge sectors or string or brane excitations. For example, the exchange of string Regge excitations of Standard Model particles in $2 \rightarrow 2$ scattering $[39,44]$ would appear as a contactlike interaction, similar to that of graviton KK exchange, but with a large strength.

It is possible that inelastic scattering at energies $\gg \mathrm{TeV}$ could be dominated by the production of strongly coupled objects such as microscopic black holes [51]. Assuming that these decay via Hawking radiation, they would then be observable in future very high-energy colliders [52].

\section{3. $\mathrm{TeV}^{-}$1-Sized Extra Dimensions}

The possibility of $\mathrm{TeV}^{-1}$-sized extra dimensions naturally arises in braneworld theories $[9,53]$. By themselves, they do not allow for a reformulation of the hierarchy problem, but they may be incorporated into a larger structure in which this problem is solved. In these scenarios, the Standard Model fields are phenomenologically allowed to propagate in the bulk. This presents a wide variety of choices for model building: (i) all, or only some, of the Standard Model gauge fields exist in the bulk; (ii) the Higgs field may lie on the brane or in the bulk; (iii) the Standard Model fermions may be confined to the brane or to specific locales in the extra dimension. The phenomenological consequences of this scenario strongly depend on the location of the fermion fields. Unless otherwise noted, our discussion assumes that all of the Standard Model gauge fields propagate in the bulk.

The masses of the excitation states in the gauge boson KK towers depend on where the Higgs boson is located. If the Higgs field propagates in the bulk, the zero-mode state of the Higgs KK tower receives a vacuum expectation value (vev) which is responsible for the spontaneous breaking of the electroweak gauge symmetry. In this case, the resulting mass matrix for the states in the gauge boson KK towers is diagonal and the excitation masses are shifted by the mass of the 
gauge zero-mode, which corresponds to the Standard Model gauge field, giving

$$
m_{\vec{n}}=\left(m_{0}^{2}+\vec{n} \cdot \vec{n} / R_{c}^{2}\right)^{1 / 2} .
$$

However, if the Higgs is confined to the brane, its vev induces mixing, amongst the gauge KK states of order $\left(m_{0} R_{c}\right)^{2}$. The KK mass matrix must then be diagonalized in order to determine the excitation masses. For the case of 1 extra $\mathrm{TeV}^{-1}$-sized dimension, the coupling strength of the gauge KK states to the Standard Model fermions on the brane is $\sqrt{2} g$, where $g$ is the corresponding Standard Model gauge coupling.

We first discuss the case where the Standard Model fermions are rigidly fixed to the brane and do not feel the effects of the additional dimensions. For models in this class, precision electroweak data place strong constraints [54] on the mass of the first gauge KK excitation. Contributions to electroweak observables arise from the virtual exchange of gauge KK states and a summation over the contributions from the entire KK tower must be performed. For $D>5$, this sum is divergent. In the full higher dimensional theory, some new, as of yet unknown, physics would regularize this sum and render it finite. An example of this is given by the possibility that the brane is flexible or non-rigid [43], which has the effect of exponentially damping the sum over KK states. Due to our present lack of knowledge of the full underlying theory, the KK sum is usually terminated by an explicit cut-off, which provides a naive estimate of the magnitude of the effects.

Since the $D=5$ theory is finite, it is the scenario that is most often discussed and is sometimes referred to as the 5-dimensional Standard Model (5DSM). In this case, a global fit to the precision electroweak data including the contributions from KK gauge interactions yields [54] $m_{1} \sim R_{c}^{-1} \gtrsim 4 \mathrm{TeV}$. In addition, the KK contributions to the precision observables allow for the mass of the Higgs boson to be somewhat heavier than the value obtained in the Standard Model global fit. Given the constraint on $R_{c}$ from the precision data set, the gauge KK contributions to the anomalous magnetic moment of the muon are small [55].

Such a large mass for the first gauge KK state is beyond the direct reach at present accelerators, as well as a future $e^{+} e^{-}$linear collider. However, they can be produced as resonances at the LHC in the Drell-Yan channel provided $m_{1} \lesssim 6 \mathrm{TeV}$. Lepton colliders can indirectly observe the existence of heavy gauge KK states in the contact interaction limit via their $s$-channel exchanges. In this case the contribution of the entire KK tower must be summed, and suffers the same problems with divergences discussed above. The resulting sensitivities to the gauge KK tower in the 5DSM from direct and indirect searches at various facilities is displayed in Table 4.

We now discuss the scenario where the Standard Model fermions are localized at specific points in the extra $\mathrm{TeV}^{-1}$-sized dimensions. In this case, the 


\begin{tabular}{|c|c|}
\hline & $m_{1}$ Reach (TeV) \\
\hline Tevatron Run II 2 $\mathrm{fb}^{-1}$ & 1.1 \\
LHC 100 fb & 6.3 \\
LEP II & 3.1 \\
LC $\sqrt{s}=0.5$ TeV 500 fb-1 & 13.0 \\
LC $\sqrt{s}=1.0$ TeV 500 fb-1 & 23.0 \\
LC $\sqrt{s}=1.5$ TeV 500 fb-1 & 31.0 \\
\hline
\end{tabular}

Table 4

$95 \%$ CL search reach for the mass $m_{1}$ of the first KK gauge boson excitation [54].

fermions have narrow gaussian-like wave functions in the extra dimensions with the width of their wave function being much smaller than $R_{c}^{-1}$. The placement of the different fermions at distinct locations in the additional dimensions, along with the narrowness of their wavefunctions, can then naturally suppress [12] operators mediating dangerous processes such as proton decay. The exchange of gauge KK states in $2 \rightarrow 2$ scattering processes involving initial and final state fermions is sensitive to the placement of the fermions and can be used to perform a cartography of the localized fermions [56], i.e., measure the wavefunctions and locations of the fermions. At very large energies, it is possible that the cross section for such scattering will tend rapidly to zero since the fermions' wavefunctions will not overlap and hence they may completely miss each other in the extra dimensions [57].

Lastly, we discuss the case of universal extra dimensions [58], where all Standard Model fields propagate in the bulk, and branes need not be present. Translational invariance in the higher dimensional space is thus preserved. This results in the tree-level conservation of the $\delta$-dimensional momentum of the bulk fields, which implies that KK parity, $(-1)^{n}$, is conserved to all orders. The phenomenology of this scenario is quite different from the cases discussed above. Since KK parity is conserved, KK excitations can no longer be produced as schannel resonances; they can now only be produced in pairs. This results in a drastic reduction of the collider sensitivity to such states, with searches at the Tevatron yielding the bounds $[58,59] m_{1} \gtrsim 400 \mathrm{GeV}$ for two universal extra dimensions. The constraints from electroweak precision data are also lowered and yield similar bounds. Since the KK states are allowed to be relatively light, they can produce observable effects $[59,60]$ in loop-mediated processes, such as $b \rightarrow s \gamma, g-2$ of the muon, and rare Higgs decays. 


\section{Warped Extra Dimensions}

In this scenario, the hierarchy between the Planck and electroweak scales is generated by a large curvature of the extra dimensions $[4,5]$. The simplest such framework is comprised of just one additional spatial dimension of finite size, in which gravity propagates. The geometry is that of a 5-dimensional Anti-deSitter space $\left(\mathrm{AdS}_{5}\right)$, which is a space of constant negative curvature. The extent of the $5^{\text {th }}$ dimension is $y=\pi R_{c}$. Every slice of the $5^{\text {th }}$ dimension corresponds to a 4-d Minkowski metric. Two 3-branes, with equal and opposite tension, sit at the boundaries of this slice of $\mathrm{AdS}_{5}$ space. The Standard Model fields are constrained to the 3-brane located at the boundary $y=\pi R_{c}$, known as the TeVbrane, while gravity is localized about the opposite brane at the other boundary $y=0$. This is referred to as the Planck brane.

The metric for this scenario preserves 4-d Poincare invariance and is

$$
d s^{2}=e^{-2 k y} \eta_{\mu \nu} d x^{\mu} d x^{\nu}-d y^{2},
$$

where the exponential function of the $5^{\text {th }}$ dimensional coordinate multiplying the usual 4-d Minkowski term indicates a non-factorizable geometry. This exponential is known as a warp factor. Here, the parameter $k$ governs the degree of curvature of the $\mathrm{AdS}_{5}$ space; it is assumed to be of order the Planck scale. Consistency of the low-energy theory sets $k / \bar{M}_{\mathrm{Pl}} \lesssim 0.1$, with $\bar{M}_{\mathrm{Pl}}=M_{\mathrm{Pl}} / \sqrt{8 \pi}=$ $2.4 \times 10^{18}$ being the reduced $4-d$ Planck scale. The relation

$$
\bar{M}_{\mathrm{Pl}}^{2}=\frac{\bar{M}_{5}^{3}}{k}
$$

is derived from the 5-dimensional action and indicates that the (reduced) 5dimensional fundamental scale $\bar{M}_{5}$ is of order $\bar{M}_{\mathrm{Pl}}$. Since $k \sim \bar{M}_{5} \sim \bar{M}_{\mathrm{Pl}}$, there are no additional hierarchies present in this model.

The scale of physical phenomena as realized by a 4-dimensional flat metric transverse to the $5^{\text {th }}$ dimension is specified by the exponential warp factor. The scale $\Lambda_{\pi} \equiv \bar{M}_{\mathrm{Pl}} e^{-k R_{c} \pi}$ then describes the scale of all physical processes on the TeV-brane. With the gravitational wavefunction being localized on the Planck brane, $\Lambda_{\pi}$ takes on the value $\sim 1 \mathrm{TeV}$ providing $k R_{c} \simeq 11-12$. It has been demonstrated [61] that this value of $k R_{c}$ can be stabilized within this configuration without the fine tuning of parameters. The hierarchy is thus naturally established by the warp factor. Note that since $k R_{c} \simeq 10$ and it is assumed that $k \sim 10^{18} \mathrm{GeV}$, this is not a model with a large extra dimension.

Two parameters govern the 4-d effective theory of this scenario [62]: $\Lambda_{\pi}$ and the ratio $k / \bar{M}_{P l}$. Note that the approximate values of these parameters are known due to the relation of this model to the hierarchy problem. As in the case of large 
extra dimensions, the Feynman rules are obtained by a linear expansion of the flat metric,

$$
G_{\alpha \beta}=e^{-2 k y}\left(\eta_{\alpha \beta}+2 h_{\alpha \beta} / M_{5}^{3 / 2}\right),
$$

which for this scenario includes the warp factor multiplying the linear expansion. After compactification, the resulting KK tower states are the coefficients of a Bessel expansion with the Bessel functions replacing the Fourier series of a flat geometry due to the strongly curved space and the presence of the warp factor. Here, the masses of the KK states are $m_{n}=x_{n} k e^{-k R_{c} \pi}=x_{n} \Lambda_{\pi} k / \bar{M}_{\mathrm{Pl}}$ with the $x_{n}$ being the roots of the first-order Bessel function, i.e., $J_{1}\left(x_{n}\right)=0$. The first excitation is then naturally of order a $\mathrm{TeV}$ and the $\mathrm{KK}$ states are not evenly spaced. The interactions of the graviton KK tower with the Standard Model fields on the TeV-brane are given by

$$
\mathcal{L}=\frac{-1}{\bar{M}_{P l}} T^{\mu \nu}(x) h_{\mu \nu}^{(0)}(x)-\frac{1}{\Lambda_{\pi}} T^{\mu \nu}(x) \sum_{n=1}^{\infty} h_{\mu \nu}^{(n)}(x) .
$$

Note that the zero-mode decouples and that the couplings of the excitation states are inverse $\mathrm{TeV}$ strength. This results in a strikingly different phenomenology than in the case of large extra dimensions.

In this scenario, the principal collider signature is the direct resonant production of the spin-2 states in the graviton KK tower. To exhibit how this may appear at a collider, Figure 6 displays the cross section for $e^{+} e^{-} \rightarrow \mu^{+} \mu^{-}$as a function of $\sqrt{s}$, assuming $m_{1}=500 \mathrm{GeV}$ and varying $k / \bar{M}_{\mathrm{Pl}}$ in the range $0.01-0.05$. The height of the third resonance is greatly reduced as the higher KK excitations prefer to decay to the lighter graviton states, once it is kinematically allowed [63]. In this case, high energy colliders may become graviton factories! If the first graviton KK state is observed, then the parameters of this model can be uniquely determined by measurement of the location and width of the resonance. In addition, the spin- 2 nature of the graviton resonance can be determined from the shape of the angular distribution of the decay products. This is demonstrated in Figure 7, which displays the angular distribution of the final state leptons in Drell-Yan production, $p p \rightarrow \ell^{+} \ell^{-}$, at the LHC [64].

Searches for the first graviton KK resonance in Drell-Yan and dijet data from Run I at the Tevatron restrict [62] the parameter space of this model, as shown in Figure 8 . These data exclude larger values of $k / \bar{M}_{\mathrm{Pl}}$ for values of $m_{1}$ which are in kinematic reach of the accelerator.

Gravitons may also contribute to precision electroweak observables. A precise description of such contributions requires a complete understanding of the full underlying theory due to the non-renormalizability of gravity. However, naive estimates of the size of such effects can be obtained in an effective field theory 


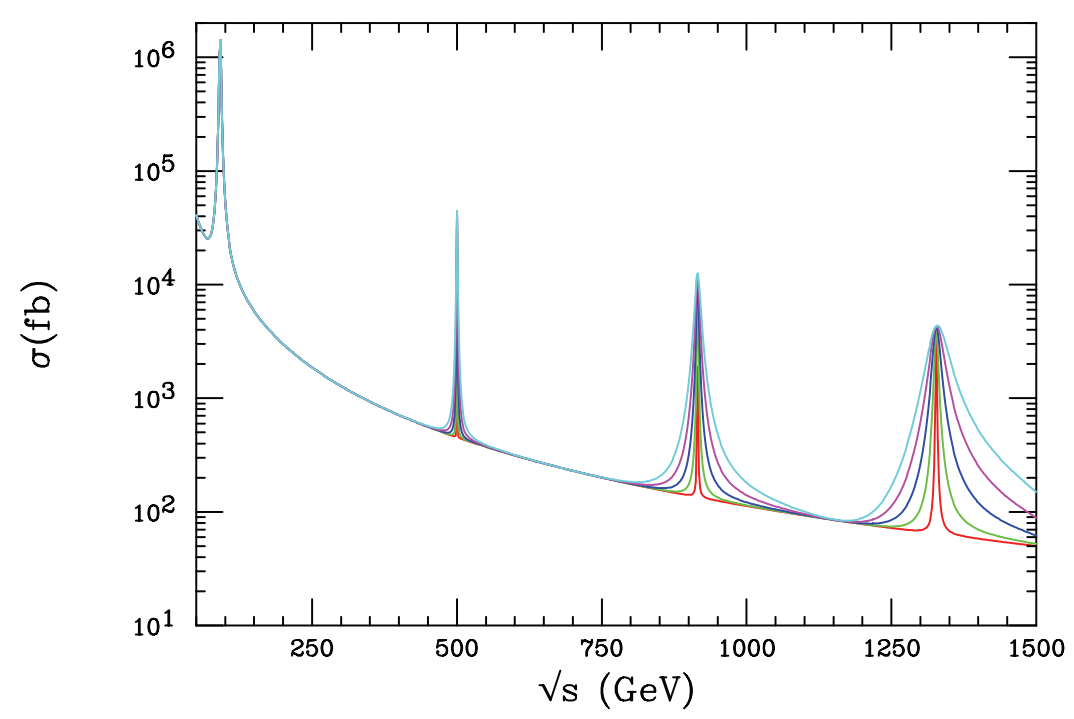

Fig. 6. The cross section for $e^{+} e^{-} \rightarrow \mu^{+} \mu^{-}$including the exchange of a KK tower of gravitons in the Randall-Sundrum model with $m_{1}=500 \mathrm{GeV}$. The curves correspond to $k / \bar{M}_{\mathrm{Pl}}=$ in the range $0.01-0.05$.

by employing a cut-off to regulate the theory [65]. The resulting cut-off dependent constraints indicate [66] that smaller values of $k / \bar{M}_{\mathrm{Pl}}$ are inconsistent with precision electroweak data, as shown in Figure 8.

These two constraints from present data, taken together with the theoretical assumptions that (i) $\Lambda_{\pi} \lesssim 10 \mathrm{TeV}$, i.e., the scale of physics on the TeV-brane is not far above the electroweak scale so that an additional hierarchy is not generated, and (ii) $k / \bar{M}_{\mathrm{Pl}} \lesssim 0.1$ from bounds on the curvature of the $\mathrm{AdS}_{5}$, result in a closed allowed region in the two parameter space. This is displayed in Figure 8, which also shows the expected search reach for resonant graviton KK production in the Drell-Yan channel at the LHC. We see that the full allowed parameter space can be completely explored at the LHC, given our theoretical prejudices, and hence the LHC will either discover or exclude this model.

If the above theoretical assumptions are evaded, the KK gravitons may be too massive to be produced directly. However, their contributions to fermion pair production may still be felt via virtual exchange. In this case, the uncertainties associated with the introduction of a cut-off are avoided, since there is only one additional dimension and the KK states may be neatly summed. The resulting sensitivities [62] to $\Lambda_{\pi}$ at current and future colliders are listed in Table 5.

The Goldberger-Wise mechanism [61] for stabilizing the separation of the 


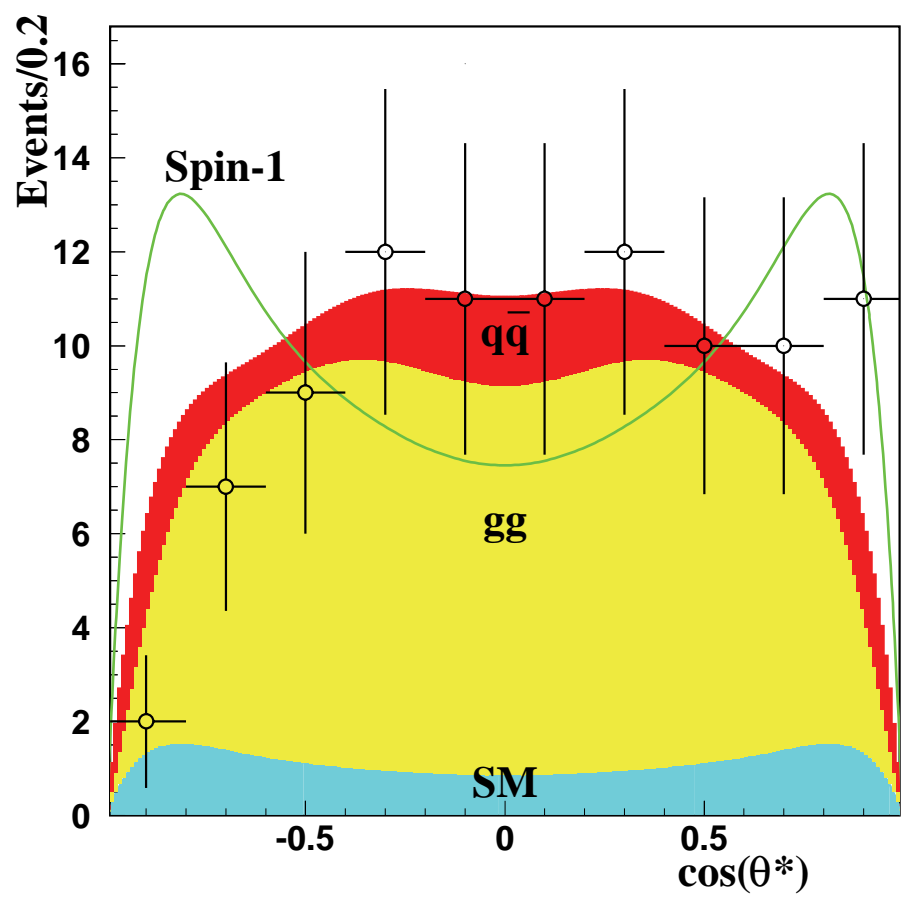

Fig. 7. The angular distribution of "data" at the LHC from Drell-Yan production of the first graviton KK excitation with $m_{1}=1.5 \mathrm{TeV}$ and $100 \mathrm{fb}^{-1}$ of integrated luminosity. The stacked histograms represent the Standard Model contributions, and $g g$ and $q \bar{q}$ initiated graviton production as labeled. The curve shows the expected distribution from a spin-1 resonance. From [64].

\begin{tabular}{|c|c|c|c|}
\hline & \multicolumn{3}{|c|}{$k / \bar{M}_{P l}$} \\
\hline & 0.01 & 0.1 & 1.0 \\
\hline LEP II & 4.0 & 1.5 & 0.4 \\
LC $\sqrt{s}=0.5 \mathrm{TeV}$ & 20.0 & 5.0 & 1.5 \\
LC $\sqrt{s}=1.0 \mathrm{TeV}$ & 40.0 & 10.0 & 3.0 \\
Tevatron Run II & 5.0 & 1.5 & 0.5 \\
LHC & 20.0 & 7.0 & 3.0 \\
\hline
\end{tabular}

Table 5

95\% CL search reach for $\Lambda_{\pi}$ in $\mathrm{TeV}$ in the contact interaction regime taking 500, 2.5, 2, and 100 $\mathrm{fb}^{-1}$ of integrated luminosity at the LC, LEP II, Tevatron, and LHC, respectively. From [62]. 


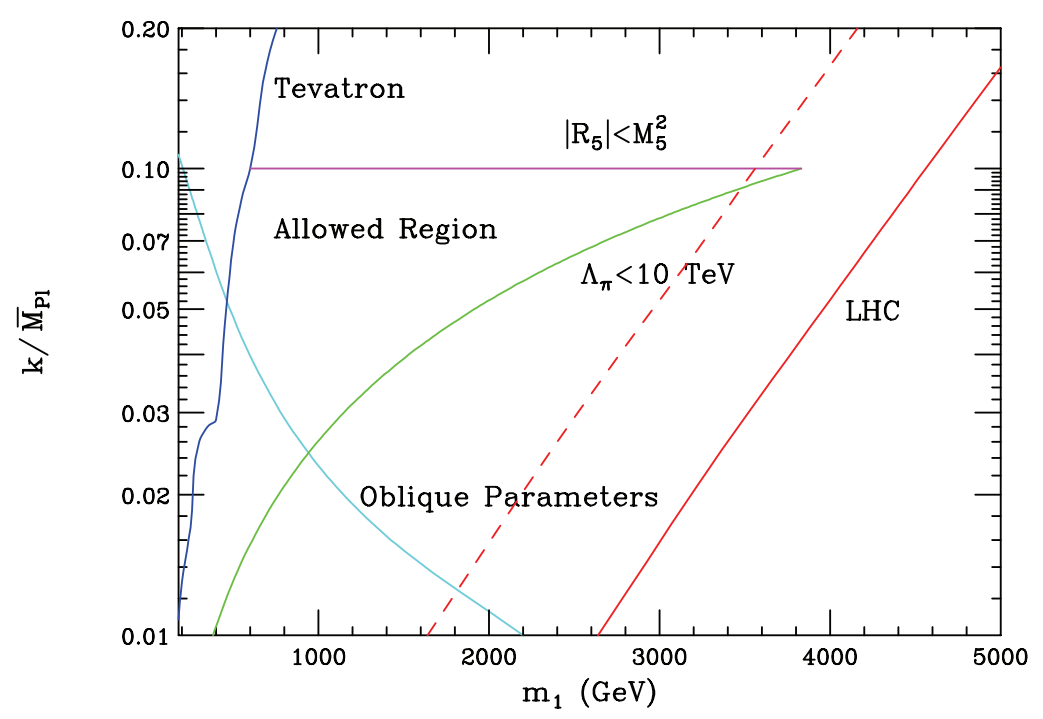

Fig. 8. Summary of experimental and theoretical constraints on the Randall-Sundrum model in the two-parameter plane $k / \bar{M}_{\mathrm{Pl}}-m_{1}$, for the case where the Standard Model fields are constrained to the TeV-brane. The allowed region lies in the center as indicated. The LHC sensitivity to graviton resonances in the Drell-Yan channel is represented by the diagonal dashed and solid curves, corresponding to 10 and $100 \mathrm{fb}^{-1}$ of integrated luminosity, respectively. From [66].

two 3-branes in this configuration with $k R_{c} \sim 10$ leads to the existence of a new, relatively light scalar field. This field is the radion and it is related to the radial fluctuations of the extra dimension, and to the scalar remnant of the bulk graviton KK decomposition. The radion couples to the Standard Model fields via the trace of the stress energy tensor with strength $\sim T_{\mu}^{\mu} / \Lambda_{\pi}$. These interactions are similar to those of the Higgs boson, and it is allowed to mix with the Higgs, which alters the couplings of both fields. The phenomenology of this field is detailed in $[14,67]$.

Astrophysical bounds are not present in this scenario since the first graviton $\mathrm{KK}$ state occurs at a $\sim \mathrm{TeV}$. However, the TeV scale graviton KK states can induce high energy cosmic rays. In this case, neutrino annihilation within a GZK distance of the earth can produce a single graviton KK state on resonance which subsequently decays hadronically [68]. For neutrinos of mass $m_{\nu} \sim 10^{-2}$ to $10^{-1} \mathrm{eV}$, and graviton resonances of order a TeV, super-GZK events can be produced. Under the assumption that the incident neutrino spectrum extends in neutrino energy with a reasonably slow fall-off, the existence of a series of $s$-channel KK graviton resonances will lead to a series of ultra-GZK events. The rates for 
these bursts are generally at or near the present level of observability for a wide range of model parameters. The fact that such events are not as yet observed can be used to constrain the parameter space of this model once a specific form of the neutrino energy spectrum is assumed.

In a variant of this model, the Standard Model fields may propagate in the bulk. This is desirable for numerous model building reasons as mentioned in the introduction. As a first step, one can study the effect of placing the Standard Model gauge fields in the bulk and keeping the fermions on the TeV-brane. In this case, one finds [69] that the fermions on the brane couple to the KK gauge fields $\sim 9$ times more strongly than they couple to the Standard Model gauge fields. This results in strong bounds on gauge KK states from their contributions to electroweak precision data. A global fit to the electroweak data set yields the constraint $m_{1} \gtrsim 25 \mathrm{TeV}$ on the first gauge $\mathrm{KK}$ mass, implying $\Lambda_{\pi} \gtrsim 100 \mathrm{TeV}$.

This bound can be relaxed if the fermions also reside in the bulk $[66,70]$. In this case, a third parameter is introduced, corresponding to the bulk fermion mass which is given by $m_{5}=\nu k$ with $\nu$ being of order one. The parameter $\nu$ controls the shape of the fermion zero mode wavefunction. The resulting phenomenology is markedly different, and is highly dependent on the parameter $\nu$. In particular, large mixing is induced between the zero-mode top-quark and the states in its KK tower. This results [71] in substantial shifts to the $\rho$-parameter and forces the third generation of fermions to be confined to the TeV-brane with only the first two generations of fermions being allowed to reside in the bulk.

An alternate scenario is possible [5] when the second brane is taken off to infinity, i.e., $R_{c} \rightarrow \infty$, and the Standard Model fields are confined to the brane at $y=0$ where gravity is localized. In this case, the graviton KK modes become continuous, i.e., the gap between KK states disappears, and their couplings to the Standard Model fields are much weaker than $M_{\mathrm{Pl}}$. This configuration no longer allows for a reformulation of the hierarchy problem, but can potentially be observable [72] in sub-mm gravitational force experiments.

Another consistent scenario of this type involves two branes, both with positive tension, separated in a five-dimensional Anti-de Sitter geometry of infinite extent [73]. The graviton is localized on one of the branes, while a gapless continuum of additional gravity modes probe the infinite fifth dimension. The phenomenological effects of this framework are similar to the process of real graviton emission in the ADD scheme with six large toroidal dimensions. The resulting cosmological constraints are also found to be very mild [74]. 


\section{Summary}

If the structure of spacetime is different than that readily observed, gravitational physics, particle physics and cosmology are all immediately affected. The physics of extra dimensions offers new insights and solutions to fundamental questions arising in these fields. Novel ideas and frameworks are continuously born and evolved. They make use of string theoretical features and tools and they may reveal if and how the 11-dimensional string theory is relevant to our four-dimensional world.

We have outlined some of the experimental observations in particle and gravitational physics as well as astrophysical and cosmological considerations thatcan constrain or confirm these scenarios. These developing ideas and the wide interdisciplinary experimental program that is charted out to investigate them mark a renewed effort to describe the dynamics behind spacetime. We look forward to the discovery of a higher dimenionsal spacetime!

\section{References}

[1] G. Nordstrom, Z. Phys. 15, 504 (1914).

[2] T. Kaluza, Preuss.Akad.Wiss, Berlin, Math. Phys. K 1, 966 (1921); O. Klein Z. Phys. 37 895; Nature 118, 516 (1926).

[3] N. Arkani-Hamed, S. Dimopoulos and G. R. Dvali, Phys. Lett. B 429, 263 (1998) [arXiv:hepph/9803315]; I. Antoniadis, N. Arkani-Hamed, S. Dimopoulos and G. R. Dvali, Phys. Lett. B 436, 257 (1998) [arXiv:hep-ph/9804398]; N. Arkani-Hamed, S. Dimopoulos and G. R. Dvali, Phys. Rev. D 59, 086004 (1999) [arXiv:hep-ph/9807344].

[4] L. Randall and R. Sundrum, Phys. Rev. Lett. 83, 3370 (1999) [arXiv:hep-ph/9905221].

[5] L. Randall and R. Sundrum, Phys. Rev. Lett. 83, 4690 (1999) [arXiv:hep-th/9906064].

[6] J. Polchinski, String Theory, Cambridge University Press (1998).

[7] N. Arkani-Hamed, S. Dimopoulos, G. R. Dvali and N. Kaloper, Phys. Rev. Lett. 84, 586 (2000) [arXiv:hep-th/9907209].

[8] K. R. Dienes, E. Dudas and T. Gherghetta, Phys. Lett. B 436, 55 (1998) [arXiv:hepph/9803466]; Nucl. Phys. B 537, 47 (1999) [arXiv:hep-ph/9806292]; Nucl. Phys. B 567, 111 (2000) [arXiv:hep-ph/9908530].

[9] I. Antoniadis, Phys. Lett. B 246, 377 (1990).

[10] D. E. Kaplan, G. D. Kribs and M. Schmaltz, Phys. Rev. D 62, 035010 (2000) [arXiv:hepph/9911293], Z. Chacko, M. A. Luty, A. E. Nelson and E. Ponton, JHEP 0001, 003 (2000) [arXiv:hep-ph/9911323]; N. Arkani-Hamed, L. J. Hall, D. R. Smith and N. Weiner, Phys. Rev. D 63, 056003 (2001) [arXiv:hep-ph/9911421].

[11] K. R. Dienes, E. Dudas and T. Gherghetta, Nucl. Phys. B 557, 25 (1999) [arXiv:hepph/9811428]; N. Arkani-Hamed, S. Dimopoulos, G. R. Dvali and J. March-Russell, Phys. Rev. D 65, 024032 (2002) [arXiv:hep-ph/9811448]; Y. Grossman and M. Neubert, Phys. Lett. B 474, 361 (2000) [arXiv:hep-ph/9912408].

[12] N. Arkani-Hamed and M. Schmaltz, Phys. Rev. D 61, 033005 (2000) [arXiv:hep-ph/9903417]. 
[13] "Flavor Physics For The Millenium”, Proceedings of the Theoretical Advanced Study Institute in Elementary Particle Physics edited by J.L. Rosner (University of Chicago) 2000.

[14] G. F. Giudice, R. Rattazzi and J. D. Wells, Nucl. Phys. B 595, 250 (2001) [arXiv:hep$\mathrm{ph} / 0002178]$.

[15] G. F. Giudice, R. Rattazzi and J. D. Wells, Nucl. Phys. B 544, 3 (1999) [arXiv:hep-ph/9811291].

[16] T. Han, J. D. Lykken and R. J. Zhang, Phys. Rev. D 59, 105006 (1999) [arXiv:hep-ph/9811350].

[17] Particle Data Group (C. Caso et al.), Eur. Phys. J. C 3, 781 (1998).

[18] D.E. Krause and E. Fischbach, arXiv:hep-ph 9912276; G.L. Smith, C.D. Hoyle, J.H. Gundlach, E.G. Adelberger, B.R. Heckel and H.E. Swanson, Phys. Rev. D 61, 022001 (2000); J. K. Hoskins, R. D. Newman, R. Spero, and J. Schultz, Phys. Rev D 32, 3084 (1985); V. P. Mitrofanov and O. I. Ponomareva, Sov. Phys. JETP 67, 1963 (1988); J.C. Long, H.W. Chan, J.C. Price, Nucl. Phys. B 539, 23 (1999); S.K. Lamoreaux, Phys. Rev. Lett. 78, 5 (1997); arXiv:quant-ph/9907076.

[19] A. H. Cook, Contemp. Phys. 28, 159 (1987).

[20] A. Kehagias and K. Sfetsos, Phys. Lett. B 472, 39 (2000) [arXiv:hep-ph/9905417].

[21] C. D. Hoyle, D. J. Kapner, B. R. Heckel, E. G. Adelberger, J. H. Gundlach, U. Schmidt and H. E. Swanson, Phys. Rev. D 70, 042004 (2004) [arXiv:hep-ph/0405262]; C. D. Hoyle, U. Schmidt, B. R. Heckel, E. G. Adelberger, J. H. Gundlach, D. J. Kapner and H. E. Swanson, Phys. Rev. Lett. 86, 1418 (2001) [arXiv:hep-ph/0011014]; E. G. Adelberger [EOT-WASH Group Collaboration], arXiv:hep-ex/0202008.

[22] N. Kaloper, J. March-Russell, G. D. Starkman and M. Trodden, Phys. Rev. Lett. 85, 928 (2000) [arXiv:hep-ph/0002001].

[23] G. R. Dvali, G. Gabadadze, M. Kolanovic and F. Nitti, Phys. Rev. D 64, 084004 (2001) [arXiv:hep-ph/0102216].

[24] K. Hirata et.al, Phys. Rev. Let. 58, 1490 (1987); R.M. Bionta et.al, Phys. Rev. Let. 58, 1494 (1987).

[25] S. Cullen and M. Perelstein, Phys. Rev. Lett. 83, 268 (1999) [arXiv:hep-ph/9903422]; V. D. Barger, T. Han, C. Kao and R. J. Zhang, Phys. Lett. B 461, 34 (1999) [arXiv:hepph/9905474]; C. Hanhart, J. A. Pons, D. R. Phillips and S. Reddy, Phys. Lett. B 509, 1 (2001) [arXiv:astro-ph/0102063].

[26] C. Hanhart, D. R. Phillips, S. Reddy and M. J. Savage, Nucl. Phys. B 595, 335 (2001) [arXiv:nucl-th/0007016].

[27] S. Hannestad and G. Raffelt, Phys. Rev. Lett. 87, 051301 (2001) [arXiv:hep-ph/0103201].

[28] S. C. Kappadath et al., Astron. Astrophys. Suppl. 120, 619 (1996); D. A. Knitten et al., Astron. Astrophys. Suppl. 120, 615 (1996); D. J. Thompson et al., Astrophys. J. Suppl. 101, 259 (1995); ibid., 107, 227 (1996); R. C. Hartman et al., Astrophys. J. Suppl. 123, 79 (1999).

[29] L. J. Hall and D. R. Smith, Phys. Rev. D 60, 085008 (1999) [arXiv:hep-ph/9904267].

[30] S. Hannestad, Phys. Rev. D 64, 023515 (2001) [arXiv:hep-ph/0102290].

[31] S. Hannestad and G. G. Raffelt, Phys. Rev. Lett. 88, 071301 (2002) [arXiv:hep-ph/0110067].

[32] See, for example, www-glast.stanford.edu.

[33] M. Fairbairn, Phys. Lett. B 508, 335 (2001) [arXiv:hep-ph/0101131].

[34] M. Fairbairn and L. M. Griffiths, JHEP 0202, 024 (2002) [arXiv:hep-ph/0111435].

[35] P. Jain, D. W. McKay, S. Panda and J. P. Ralston, Phys. Lett. B 484, 267 (2000) [arXiv:hepph/0001031]; A. Goyal, A. Gupta and N. Mahajan, Phys. Rev. D 63, 043003 (2001) [arXiv:hep$\mathrm{ph} / 0005030]$. 
[36] E. A. Mirabelli, M. Perelstein and M. E. Peskin, Phys. Rev. Lett. 82, 2236 (1999) [arXiv:hepph/9811337]; K. M. Cheung and W. Y. Keung, Phys. Rev. D 60, 112003 (1999) [arXiv:hep$\mathrm{ph} / 9903294]$.

[37] For a summary of LEP results on graviton emission, see, G. Landsberg, arXiv:hep-ex/0105039. See also, P.Abreu et al., The DELPHI Collaboration, Eur. Phys. J. C 17, 53 (2000); G. Abbiendi et al., The OPAL Collaboration, Eur. Phys. J. C 18, 253 (2000); M.Acciari et al., The L3 collaboration, Phys. Lett. B 464, 135 (1999).

[38] J. A. Aguilar-Saavedra et al. [ECFA/DESY LC Physics Working Group Collaboration], TESLA Technical Design Report, Part III, Editors R.D. Heuer, D.J. Miller, F. Richard, P.M. Zerwas, 2001, arXiv:hep-ph/0106315.

[39] S. Cullen, M. Perelstein and M. E. Peskin, Phys. Rev. D 62, 055012 (2000) [arXiv:hep$\mathrm{ph} / 0001166]$.

[40] L. Vacavant and I. Hinchliffe, J. Phys. G 27, 1839 (2001).

[41] H. Murayama and J. D. Wells, Phys. Rev. D 65, 056011 (2002) [arXiv:hep-ph/0109004].

[42] J. L. Hewett, Phys. Rev. Lett. 82, 4765 (1999) [arXiv:hep-ph/9811356].

[43] M. Bando, T. Kugo, T. Noguchi and K. Yoshioka, Phys. Rev. Lett. 83, 3601 (1999) [arXiv:hep$\mathrm{ph} / 9906549]$.

[44] E. Dudas and J. Mourad, Nucl. Phys. B 575, 3 (2000) [arXiv:hep-th/9911019]; E. Accomando, I. Antoniadis and K. Benakli, Nucl. Phys. B 579, 3 (2000) [arXiv:hep-ph/9912287].

[45] E. Eichten, K. D. Lane and M. E. Peskin, Phys. Rev. Lett. 50, 811 (1983).

[46] ALEPH Collaboration, ALEPH Note CONF-2000-005; DELPHI Collaboration, DELPHICONF-464 (2001); L3 Collaboration, L3 Note 2650 (2001); OPAL Collaboration, OPAL Note PN469 (2001); D. Bourilkov, arXiv:hep-ex/0103039.

[47] B. Abbott et al., D0 Collaboration, Phys. Rev. Lett. 86, 1156 (2001).

[48] C. Adloff et al., Phys. Lett. B 479, 358 (2000); A. Zarnecki, presented at Workshop on Higgs and Supersymmetry, Orsay, France, March 2001.

[49] F. Gianotti et al., arXiv:hep-ph/0204087; A. Miagkov, talk presented at Workshop on Higgs and Supersymmetry, Orsay, France, March 2001.

[50] T. G. Rizzo, Phys. Rev. D 60, 115010 (1999) [arXiv:hep-ph/9904380]; H. Davoudiasl, Phys. Rev. D 60, 084022 (1999) [arXiv:hep-ph/9904425]; Phys. Rev. D 61, 044018 (2000) [arXiv:hep-ph/9907347].

[51] S. B. Giddings and S. Thomas, Phys. Rev. D 65, 056010 (2002) [arXiv:hep-ph/0106219]; S. Dimopoulos and G. Landsberg, Phys. Rev. Lett. 87, 161602 (2001) [arXiv:hep-ph/0106295]; M. B. Voloshin, Phys. Lett. B 518, 137 (2001) [arXiv:hep-ph/0107119].

[52] T. G. Rizzo, JHEP 0202, 011 (2002) [arXiv:hep-ph/0201228].

[53] J. D. Lykken, Phys. Rev. D 54, 3693 (1996) [arXiv:hep-th/9603133]; I. Antoniadis and M. Quiros, Phys. Lett. B 392, 61 (1997) [arXiv:hep-th/9609209].

[54] T. G. Rizzo and J. D. Wells, Phys. Rev. D 61, 016007 (2000) [arXiv:hep-ph/9906234]; M. Masip and A. Pomarol, Phys. Rev. D 60, 096005 (1999) [arXiv:hep-ph/9902467]; W. J. Marciano, Phys. Rev. D 60, 093006 (1999) [arXiv:hep-ph/9903451].

[55] P. Nath and M. Yamaguchi, Phys. Rev. D 60, 116006 (1999) [arXiv:hep-ph/9903298].

[56] T. G. Rizzo, Phys. Rev. D 64, 015003 (2001) [arXiv:hep-ph/0101278].

[57] N. Arkani-Hamed, Y. Grossman and M. Schmaltz, Phys. Rev. D 61, 115004 (2000) [arXiv:hep$\mathrm{ph} / 9909411]$.

[58] T. Appelquist, H. C. Cheng and B. A. Dobrescu, Phys. Rev. D 64, 035002 (2001) [arXiv:hep$\mathrm{ph} / 0012100]$. 
[59] T. G. Rizzo, Phys. Rev. D 64, 095010 (2001) [arXiv:hep-ph/0106336].

[60] K. Agashe, N. G. Deshpande and G. H. Wu, Phys. Lett. B 514, 309 (2001) [arXiv:hepph/0105084]; T. Appelquist and B. A. Dobrescu, Phys. Lett. B 516, 85 (2001) [arXiv:hepph/0106140]; F. J. Petriello, arXiv:hep-ph/0204067.

[61] W. D. Goldberger and M. B. Wise, Phys. Rev. Lett. 83, 4922 (1999) [arXiv:hep-ph/9907447].

[62] H. Davoudiasl, J. L. Hewett and T. G. Rizzo, Phys. Rev. Lett. 84, 2080 (2000) [arXiv:hep$\mathrm{ph} / 9909255]$.

[63] H. Davoudiasl and T. G. Rizzo, Phys. Lett. B 512, 100 (2001) [arXiv:hep-ph/0104199].

[64] B. C. Allanach, K. Odagiri, M. A. Parker and B. R. Webber, JHEP 0009, 019 (2000) [arXiv:hep$\mathrm{ph} / 0006114]$.

[65] For a discussion of this point, see, R. Contino, L. Pilo, R. Rattazzi and A. Strumia, JHEP 0106, 005 (2001) [arXiv:hep-ph/0103104].

[66] H. Davoudiasl, J. L. Hewett and T. G. Rizzo, Phys. Rev. D 63, 075004 (2001) [arXiv:hep$\mathrm{ph} / 0006041]$.

[67] W. D. Goldberger and M. B. Wise, Phys. Lett. B 475, 275 (2000) [arXiv:hep-ph/9911457]; K. M. Cheung, Phys. Rev. D 63, 056007 (2001) [arXiv:hep-ph/0009232]; J. L. Hewett and T. G. Rizzo, arXiv:hep-ph/0202155.

[68] H. Davoudiasl, J. L. Hewett and T. G. Rizzo, arXiv:hep-ph/0010066.

[69] H. Davoudiasl, J. L. Hewett and T. G. Rizzo, Phys. Lett. B 473, 43 (2000) [arXiv:hepph/9911262]; A. Pomarol, Phys. Lett. B 486, 153 (2000) [arXiv:hep-ph/9911294].

[70] S. Chang, J. Hisano, H. Nakano, N. Okada and M. Yamaguchi, Phys. Rev. D 62, 084025 (2000) [arXiv:hep-ph/9912498]; T. Gherghetta and A. Pomarol, Nucl. Phys. B 586, 141 (2000) [arXiv:hep-ph/0003129].

[71] J. L. Hewett, F. J. Petriello and T. G. Rizzo, arXiv:hep-ph/0203091.

[72] D. J. Chung, L. L. Everett and H. Davoudiasl, Phys. Rev. D 64, 065002 (2001) [arXiv:hep$\mathrm{ph} / 0010103]$.

[73] J. Lykken and L. Randall, JHEP 0006, 014 (2000) [arXiv:hep-th/9908076].

[74] A. Hebecker and J. March-Russell, Nucl. Phys. B 608, 375 (2001) [arXiv:hep-ph/0103214]. 